University of Wollongong

Research Online

Faculty of Engineering and Information

Faculty of Engineering and Information

Sciences - Papers: Part B

Sciences

2019

\title{
Modelling of the Silicon-On-Insulator microdosimeter response within the International Space Station for astronauts' radiation protection
}

\author{
Stefania Peracchi \\ University of Wollongong, sp009@uowmail.edu.au \\ James Vohradsky \\ University of Wollongong, jev720@uowmail.edu.au \\ Susanna Guatelli \\ University of Wollongong, susanna@uow.edu.au \\ David Bolst \\ University of Wollongong, dbolst@uow.edu.au \\ Linh T. Tran \\ University of Wollongong, tttran@uow.edu.au
}

See next page for additional authors

Follow this and additional works at: https://ro.uow.edu.au/eispapers1

Part of the Engineering Commons, and the Science and Technology Studies Commons

Research Online is the open access institutional repository for the University of Wollongong. For further information contact the UOW Library: research-pubs@uow.edu.au 


\title{
Modelling of the Silicon-On-Insulator microdosimeter response within the International Space Station for astronauts' radiation protection
}

\begin{abstract}
Astronauts are exposed to high-energy cosmic radiation which may have harmful health effects. At the altitude of the International Space Station (ISS), the main radiation sources are Galactic Cosmic Rays (GCRs), Solar Particle Events (SPEs) and trapped protons of the Van Allen Belts. The radiation field mainly consists of protons, helium nuclei and heavy ions with energies up to hundreds of $\mathrm{GeV} / \mathrm{n}$. A powerful approach to determine the effect of space radiation on astronauts is microdosimetry. The Centre for Medical Radiation Physics is active in the development of Silicon-On-Insulator (SOI) microdosimeters, as an alternative to Tissue Equivalent Proportional Counters (TEPCs) for radiation protection purposes. SOI microdosimeters are portable and do not require a high-voltage power supply. They consist of a matrix of silicon Sensitive Volumes (SV), which mimic the dimensions of biological cells. In this study, we investigated for the first time the response of the 3D "Mushroom" microdosimeter, a type of SOI microdosimeter in the Columbus module of the ISS. Tissue-equivalent microdosimetric spectra of GCRs, SPEs, and trapped protons were obtained to estimate the dose equivalent delivered to the astronauts. Results demonstrate a non-negligible production of secondary particles due to the propagation of space radiation through the wall of the Columbus and the microdosimeter. A number of heavy ions were detected with high lineal energies, these events pose a significant hazard in terms of radiation protection. Moreover, the dose evaluation shows a good agreement with experimental data found in the literature, confirming the suitability of our Geant4 model and the feasibility of using the SOI microdosimeter for ISS astronauts' personal dosimetry.

\section{Disciplines}

Engineering | Science and Technology Studies

\section{Publication Details}

Peracchi, S., Vohradsky, J., Guatelli, S., Bolst, D., Tran, L. T., Prokopovich, D. A. \& Rosenfeld, A. B. (2019). Modelling of the Silicon-On-Insulator microdosimeter response within the International Space Station for astronauts' radiation protection. Radiation Measurements, 128 106182-1-106182-7.
\end{abstract}

\section{Authors}

Stefania Peracchi, James Vohradsky, Susanna Guatelli, David Bolst, Linh T. Tran, Dale A. Prokopovich, and Anatoly B. Rosenfeld 


\title{
Modelling of the Silicon-On-Insulator microdosimeter response within the International Space Station for astronauts' radiation protection
}

\author{
S. Peracchi ${ }^{1, *}$, J. Vohradsky ${ }^{1}$, S. Guatelli ${ }^{1}$, D. Bolst ${ }^{1}$, L. T. Tran ${ }^{1}$, D. A. Prokopovich ${ }^{2}$ and A. B. Rosenfeld ${ }^{1}$. \\ ${ }^{l}$ Center for Medical Radiation Physics, University of Wollongong, Wollongong NSW Australia \\ ${ }^{2}$ Australia's Nuclear Science and Technology Organization, Lucas Heights NSW Australia \\ *Corresponding author: email sp009@uowmail.edu.au
}

\begin{abstract}
Astronauts are exposed to high-energy cosmic radiation which may have harmful health effects. At the altitude of the International Space Station (ISS), the main radiation sources are Galactic Cosmic Rays (GCRs), Solar Particle Events (SPEs) and trapped protons of the Van Allen Belts. The radiation field mainly consists of protons, helium nuclei and heavy ions with energies up to hundreds of $\mathrm{GeV} / \mathrm{n}$. A powerful approach to determine the effect of space radiation on astronauts is microdosimetry. The Centre for Medical Radiation Physics is active in the development of Silicon-On-Insulator (SOI) microdosimeters, as an alternative to Tissue Equivalent Proportional Counters (TEPCs) for radiation protection purposes. SOI microdosimeters are portable and do not require a high-voltage power supply. They consist of a matrix of silicon Sensitive Volumes (SV), which mimic the dimensions of biological cells.

In this study, we investigated for the first time the response of the 3D "Mushroom" microdosimeter, a type of SOI microdosimeter in the Columbus module of the ISS. Tissue-equivalent microdosimetric spectra of GCRs, SPEs, and trapped protons were obtained to estimate the dose equivalent delivered to the astronauts. Results demonstrate a non-negligible production of secondary particles due to the propagation of space radiation through the wall of the Columbus and the microdosimeter. A number of heavy ions were detected with high lineal energies, these events pose a significant hazard in terms of radiation protection.

Moreover, the dose evaluation shows a good agreement with experimental data found in the literature, confirming the suitability of our Geant 4 model and the feasibility of using the SOI microdosimeter for ISS astronauts' personal dosimetry.
\end{abstract}

Keywords - Cosmic radiation, ISS, radiation protection, SOI microdosimetry, Geant4

\section{INTRODUCTION}

Astronauts are exposed to high energy cosmic radiation, which may have harmful health effects. At the altitude of the International Space Station (ISS), there are three main radiation sources. Galactic Cosmic Rays (GCRs) are a constant component of the radiation field made up of approximately $87 \%$ protons, $12 \%$ helium nuclei and $1 \%$ heavy ions (e.g. $\mathrm{C}, \mathrm{O}, \mathrm{Si}, \mathrm{Fe}$ ) with a wide energy range, up to hundreds of $\mathrm{GeV} / \mathrm{n}$. Solar Particle Events (SPEs) mainly consist of protons emitted by the Sun during periods of intense activity, with energies up to hundreds of $\mathrm{MeV}$ [1]. Because of the Earth magnetic field, protons and electrons are trapped in the Van Allen Belts.

Recent research predicts that the cosmic rays exposure during long space missions outside the Earth's magnetosphere, as to Mars, can double the cancer risk [2]. It is therefore paramount to characterize cosmic radiation and its effects on astronauts' health. A powerful approach to determine the effect of space radiation in astronauts is microdosimetry [3]. Tissue Equivalent Proportional Counters (TEPCs) are currently the standard for experimental microdosimetry. TEPCs have a large spherical volume suitable for low particles fluxes and filled with tissue-equivalent gas. Nevertheless, TEPCs have relatively large dimensions which reduce the spatial resolution and introduce wall effects. In addition, the system complexity requires a high voltage supply [4].

The Centre for Medical Radiation Physics (CMRP), University of Wollongong, is active in the development of Silicon-On-Insulator (SOI) microdosimeters, as an alternative to TEPCs, for radiation protection purposes [5-8]. With respect to conventional TEPCs, the proposed SOI microdosimeters are portable and do not require a high voltage power supply. SOI microdosimeters consists of a matrix of silicon Sensitive Volumes (SV), which mimick a cell distribution $[5,9,10]$. 
The new 3D Sensitive Volume (SV) microdosimeter called "Mushroom" is investigated in this work [11], with the intent to characterize, for the first time, its response in the radiation environment typical of the ISS. Here we report the results emerging from the characterization of the radiation environment inside and outside the Columbus module of the ISS, and the microdosimetric response of the " $3 \mathrm{D}$ Mushroom", by means of Monte Carlo simulations.

\section{MATERIALS AND METHODS}

The study was performed by means of Geant4-based simulations [11]. In order to reduce the execution times involved with the project, the simulation study consisted of two parts. The first one (described in section A) intended to characterize the radiation environment inside the Columbus ISS module. The second one (described in section B) modelled the response of a Mushroom device when exposed to the radiation environment calculated by the first study.

\section{A. Characterization of the space radiation field at the ISS altitude}

The first goal of this study was the accurate characterization of the radiation environment inside the ISS, in the Low Earth Orbit. The SPENVIS online software tool [12] was adopted to obtain the cosmic radiation spectra of the four main radiation sources outside the ISS. A mission of two days was considered with an orbit at $408 \mathrm{~km}$ apogee and $401 \mathrm{~km}$ perigee, with ISS's inclination of $51.6^{\circ}$. The following models were selected to describe the radiation field surrounding the ISS:

- $\quad$ the NASA AP8/AE8 models for trapped protons and electrons, with an energy range up to 400 $\mathrm{MeV}$ and $7 \mathrm{MeV}$, respectively;

- the CREME96 model for GCRs protons and helium nuclei with a maximum kinetic energy of $100 \mathrm{GeV} / \mathrm{n}$; the energy spectra are for a solar minimum, corresponding to the worst case scenario for the GCRs;

- the JPL-91 model for SPE protons with kinetic energy up to $500 \mathrm{MeV}$.

It is well known that the region of the South Atlantic Anomaly presents an anisotropy regarding the trapped particles flux. Several approaches can be adopted due to the existence of several models used to mimic the radiation environments of trapped particles. We decided to use the AP8/AE8 model as it is the NASA de facto standard for mission planning. It is one of the models considering an isotropic flux together with the PSB97 and the CRRESPRO models. An advance extension of the AP8/AE8 model, based on the Badhwar \& Konradi 1990 model called UP [13], allows taking into account the anisotropy. Nevertheless, the study performed by (T. Ersmark et al., 2007) investigating the difference in using the above-cited different models, shows that dose rates due to incident anisotropic trapped particles are lower, or equal to those of omnidirectional models and the AP8/AE8 model is the more favourably comparable to experimental data between all isotropic models [11].

We adopted models for solar minimum conditions, which represent the worst case scenario: the solar cycle lasts 11 years and is characterized by phases of maximum and minimum intensity. It is well known that the GCR flux is anti-correlated to the solar activity [14].

In the Geant 4 simulation, the radiation field was modeled by means of the General Particle Source (GPS), as emerging from a sphere $S_{1}$ with radius $R_{1}=1 \mathrm{~km}$, towards the ISS. The European laboratory Columbus was modelled in the simulation at the center of $S_{1}$ (see Figure 1a-b). The direction of the incident radiation was modeled with a cosine distribution. This configuration of generation of primary particles is appropriate to model the isotropic radiation field typical of outer space when the radius of $\mathrm{S}_{1}$ is significantly larger than the size of the ISS like in our simulation study [11]. In order to reduce the execution times of the simulation, particles were modeled emerging from a cone with a half-aperture angle $\theta_{\max }$, which is the maximum angle that subtends the entire Columbus (see the grey area in Figure $1 \mathrm{a}-\mathrm{b})$.

The Columbus module was modeled as a cylinder with sizes $5 \mathrm{~m}$ in diameter and $8 \mathrm{~m}$ in height, set in the center of the $S_{1}$. Based on specifications of the real Columbus' structure and materials [15], the walls of the cylinder were modeled as a multilayer of different materials (e.g. Aluminum, Kevlar and Nextel) and thicknesses (Figure 1c). 
Geant4 10.4 was used and the following Physics constructors were adopted to model all physics interactions: G4EmStandardPhysics_option3, G4HadronPhysicsQGSP_BIC_HP G4HadronElasticPhysicsHP, G4EmExtraPhysics, G4StoppingPhysics, G4IonBinaryCascadePhysics, G4RadioactiveDecayPhysics, and G4DecayPhysics.

The kinetic energy spectra of all primary and secondary particles produced within the multilayer were retrieved as output of the simulation when reaching the inner habitat of the Columbus, as shown in Figure 1. Due to simulating a cone source with a half-aperture angle of $\theta_{\max }$, to obtain the real counts $C_{r}$ of the particles reaching the inner habitat of the Columbus, the simulation results $C_{s}$ were scaled based on the formula [16]:

$$
\mathrm{C}_{\mathrm{r}}=\mathrm{C}_{\mathrm{s}} * \frac{\mathrm{N}_{\mathrm{r}}}{\mathrm{N}_{\mathrm{s}}}
$$

where $N_{s}$ is the number of simulated events. $N_{r}$ is the number of real events expected, calculated by the formula:

$$
\mathrm{N}_{\mathrm{r}}=\phi * 4 \pi^{2} * \mathrm{R}_{1}^{2} * \sin ^{2} \theta_{\max },
$$

where $\phi$ is the total energy integrated flux of incident particles simulated by SPENVIS in outer space.
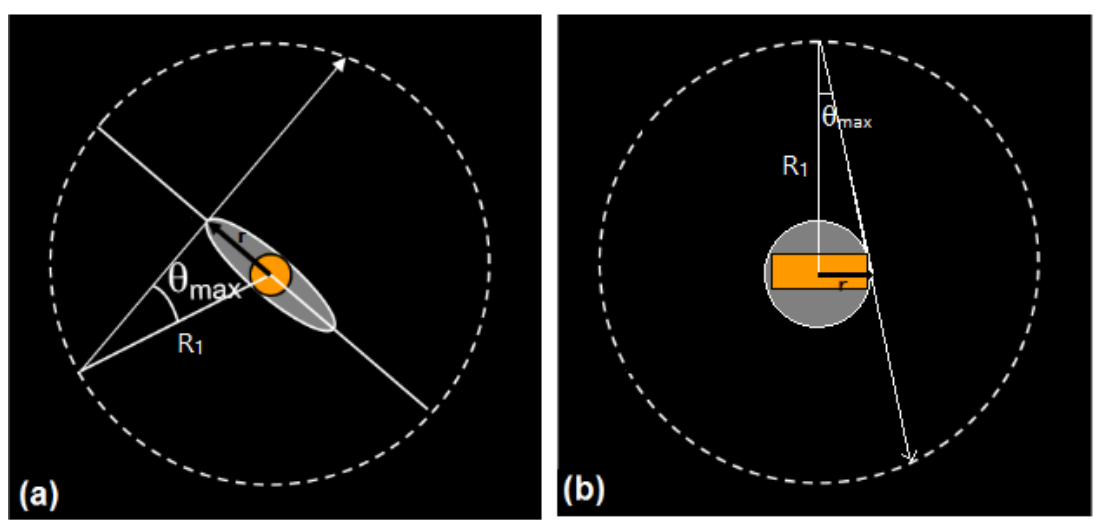

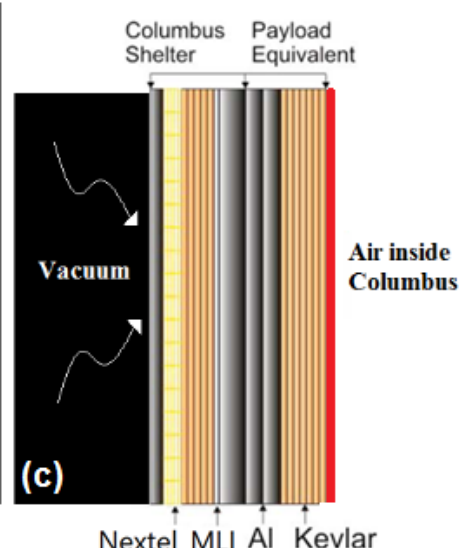

Nextel MLI Al Kevlar

Figure 1. Model of the Geant4 simulation set-up, with the space radiation components (GCR, SPE, and trapped radiation) generated from random points on the sphere $\mathrm{S}_{1}$ (dashed line). The radiation is incident on the ISS/Columbus module, represented by the orange shape (frontal view of the cylinder ((a) frontal view of the cylinder and (b) lateral view of the same). (c) Geant4 model of the Columbus multilayer: the red line represents the surface where the output radiation flux is retrieved [14].

\section{B. Characterization of the response of the $3 D$ Mushroom microdosimeter}

The energy spectra of particles recorded after the Columbus wall in the simulation study described in section A were then used to model the radiation field inside the Columbus. The particles were generated as emerging from a sphere $S_{2}$ with radius $R_{2}$ equal to the radius of the innermost cylinder volume (astronauts' habitat) of the Columbus. They were generated with an isotropic distribution inside the Columbus by means of a cosine distribution of the direction. The microdosimeter is set in the center of the sphere $S_{2}$, which corresponds also to the center of the Columbus. We adopted the same biasing method of the angular distribution (analogously to the first part of the study), to increase the number of particles interacting with the microdosimeter.

Figure 2 shows the Geant 4 model of the simulated 3D mushroom detector. The design of the 3D Mushroom is characterized by an array of 2500 cylindrical SVs where each consists of an $\mathrm{n}^{+}$core electrode surrounded by a ring $\mathrm{p}^{+}$electrode. The SV has dimensions of $30 \mu \mathrm{m}$ in diameter and $10 \mu \mathrm{m}$ in height. They are embedded in PolyMethylMethAcryl (PMMA) and placed on a $0.85 \mu \mathrm{m}$ thick $\mathrm{SiO}_{2}$ deposited above $300 \mu \mathrm{m}$ support wafer. [17-19].

We considered the microdosimeter surrounded by a PMMA sphere of $10 \mathrm{~mm}$ radius. 

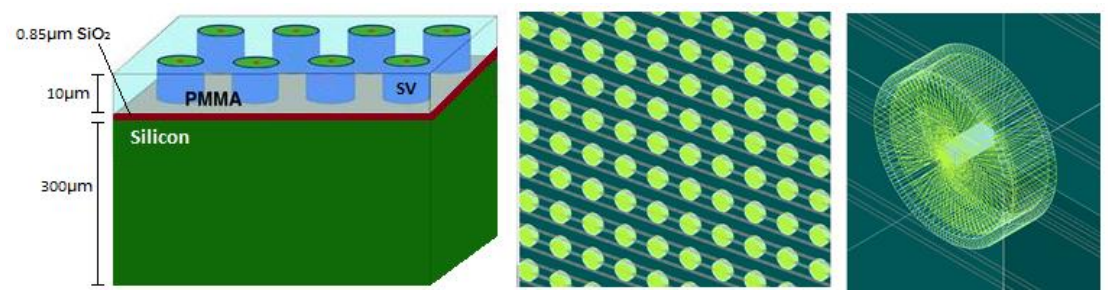

Figure 2. From the left: the 3D "Mushroom" microdosimeter design; the Geant4 SVs array; the zoomed view of a SV.

The output of the simulation is the frequency of the energy deposition $E$ of each single event occurring in the silicon SVs of the microdosimeter. The lineal energy $y$ was then calculated with the following formula:

$$
\mathrm{y}=\frac{\mathrm{E}}{\langle 1\rangle},
$$

where $\langle l\rangle$ is the mean chord length of the SV calculated by the Cauchy's formula in isotropic fields

$$
\langle 1\rangle=\frac{4 \mathrm{~V}}{\mathrm{~S}},
$$

where $\mathrm{V}$ and $\mathrm{S}$ are the volume and the total area of the SV, respectively.

To obtain the tissue equivalent dose, $D_{T E}$ from the Mushroom, the silicon microdosimetric response in terms of lineal energy spectrum $y d(y)$ [3] was converted to tissue equivalent as follows:

$$
\mathrm{D}_{\mathrm{TE}}=\mathrm{D}_{\mathrm{Si}} * 0.58,
$$

where the Absorbed Dose in silicon $D_{S i}$ is multiplied by the Tissue Equivalent factor 0.58 obtained from tissue equivalency studies for mixed fields [20,21].

Finally, the Dose Equivalent $H$ is calculated with the following formula:

$$
\mathrm{H}=\mathrm{D}_{\mathrm{TE}} * \mathrm{Q}_{\text {mean }} \text {, }
$$

where $Q_{\text {mean }}$ is the mean value of the Quality Factor derived based on the convolution of the d(y) with distribution $Q(y)$ defined by the ICRU Report 40 [22].

$$
\begin{gathered}
\mathrm{Q}_{\text {mean }}=\int_{0}^{\infty} Q(y) d(y) d y \\
\mathrm{Q}(\mathrm{y})=\frac{\mathrm{a}_{1}}{\mathrm{y}} \mathrm{e}^{\left(-\mathrm{a}_{2} * \mathrm{y}^{2}-\mathrm{a}_{3} * \mathrm{y}^{3}\right)}
\end{gathered}
$$

with coefficients $\mathrm{a}_{1}=5510 \mathrm{keV} / \mu \mathrm{m}, \mathrm{a}_{2}=5 \cdot 10^{-5} \mu \mathrm{m}^{2} / \mathrm{keV}^{2}$, and $\mathrm{a}_{3}=2 \cdot 10^{-7} \mu \mathrm{m}^{3} / \mathrm{keV}^{3}$.

In the Report 40, the liaison group of ICRP and ICRU recommends to increase the quality factors for densely ionizing radiations, such as neutrons, but also to assign quality factors less than unity to sparsely ionizing radiations, such as $\mathrm{X}$ rays or fast electrons. Despite newer definitions of the quality factor as a function of LET, our choice of adopting the definition of $Q$ in function of $y$ was due to the fact that the SOI microdosimeter can directly measure the lineal energy $y$ for each single event occurring in the SV. Compared to the ICRP-60 definition of $Q(L E T)$ approximated to 1 for $L E T<10 \mathrm{keV} / \mu \mathrm{m}$ [23], SOI microdosimters allow lineal energy measurements as low as $0.15 \mathrm{keV} / \mu \mathrm{m}$ [24] leading to a direct measurement of $Q(y)<1$ at low $y$, according to Equation 8. This experimental threshold was adopted in the simulation.

The interest was in evaluating the daily dose delivered to astronauts in order to compare it to the dose rate measured during experimental campaigns on the ISS reported in the literature. 
For each radiation source (GCR, SPE and trapped radiation), $2 \cdot 10^{9}$ primary particles ( $\left.N_{\text {simulated }}\right)$ were simulated. Integrating the initial differential flux $\phi_{i}$ inside the Columbus over all energies $E_{i}$, we could estimate $N_{1 \text { day }}$, the real number of incident particles entering the Columbus in 24 hours, as follow:

$$
\mathrm{N}_{1 \text { day }}=\left(\sum_{\text {All energies }} \phi_{\mathrm{i}} * \mathrm{E}_{\mathrm{i}}\right) * \mathrm{~S}_{\text {Columbus }} * 2 \pi * 86400 \text {, }
$$

where the total integrated flux was multiplied by the surface of the Columbus module $S_{\text {Columbus }}, 86400$ seconds in a day and $2 \pi$, assuming that only half of the Columbus module of the ISS is facing the incoming cosmic field from outer space, because the other half is facing the Earth.

The dose simulated was normalized per incident particles and then scaled for the number of particles expected in 1 day:

$$
\text { Dose }_{1 \text { day }}=\text { Dose }_{\text {simulated }} * \frac{\mathrm{N}_{\text {lday }}}{\mathrm{N}_{\text {simulated }}} \text {. }
$$

\section{RESULTS}

\section{A. Characterization of the radiation environment outside and inside the Columbus module of ISS}

The energy spectra of the radiation environment outside the Columbus, modeled with SPENVIS, are shown in Figure 3 (green lines), with their corresponding spectra modeled inside the Columbus with Geant4 (solid lines). As expected, incident GCR protons and helium nuclei with energy above $10 \mathrm{GeV} / \mathrm{n}$ are not stopped by the multilayer wall and they reach the inside of the spacecraft where astronauts live and work. Particles with energy above $1 \mathrm{GeV} / \mathrm{u}$ are slowed down during the propagation through the multilayer, with a reduction of one order of magnitude to the differential flux, while protons below 5 $\mathrm{MeV}$ are fully absorbed. It can be observed that the space radiation produces high fluxes of neutrons from spallation reactions and a non-negligible flux of nuclear products with energy of hundreds of $\mathrm{MeV} / \mathrm{n}$. Similar results were obtained for SPE protons. After the Columbus' wall, the majority of trapped protons have energies above $100 \mathrm{MeV}$, accompanied by a significant production of secondary low energy neutrons. Because of their low energy (up to $7 \mathrm{MeV}$ ), all trapped electrons are stopped during the propagation through the Columbus' wall. The figures also show that the main secondary particles' components are protons, helium nuclei and neutrons. In addition, minor contributions from other secondary particles, like gamma rays, electrons and nuclear fragments produced during the propagation through the wall, were modeled and included in the radiation environment incident on the microdosimeter. 

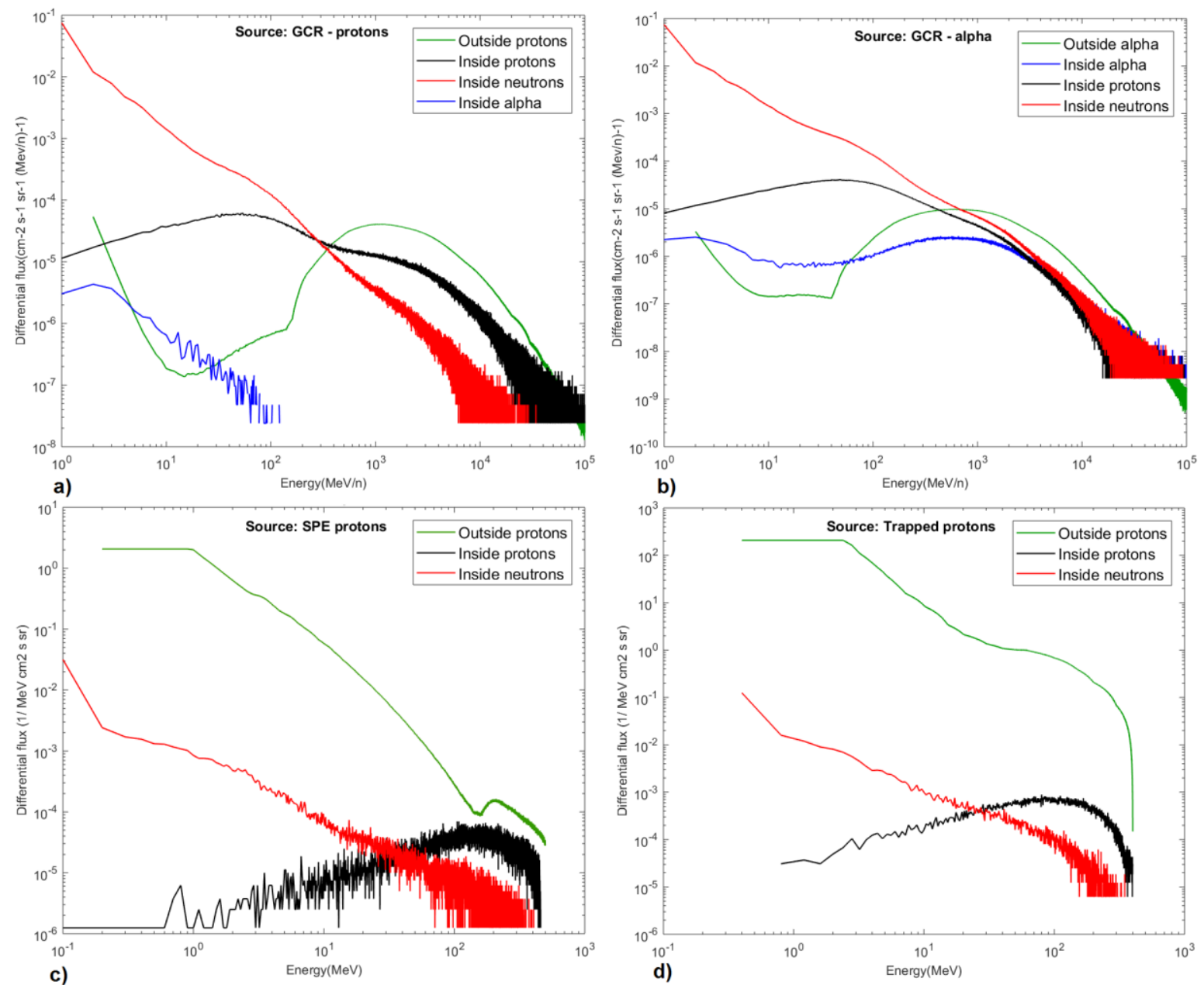

Figure 3. Simulated spectra inside the ISS behind the multilayer wall for main sources: a) GCR protons, b) GCR alpha, c) SPE protons and d) Trapped protons. The green lines represent the radiation spectra outside the ISS obtained from SPENVIS and used as input to the simulation. The solid lines are particles inside the ISS: the blue lines represent helium nuclei, the black lines are protons and the red lines are secondary neutrons.

\section{B. Response of the 3D Mushroom microdosimeter}

We simulated the response of one 3D Mushroom microdosimeter: this decision was made in order to be able to evaluate which is the number of microdosimeters to have an acceptable counts statistics during a 1day mission in space.

The MCA spectra in Figure 4 show the frequency of energy deposition events for the 7 most abundant particle species recorded by the microdosimeter. From the zoom of the spectra up to $6 \mathrm{MeV}$ we can definitely identify an abundance of particles depositing energy up to $3 \mathrm{MeV}$. The total number of counts per day detected is in a range of $10^{5} \div 10^{7}$, with the exception of protons that are above $10^{8}$ counts/day. However, we found that helium nuclei mainly produced by protons deposit energy up to $6.4 \mathrm{MeV}$ and ${ }^{12} \mathrm{C}$ ions up to $7.3 \mathrm{MeV}$. Several other heavier ions as ${ }^{13} \mathrm{C},{ }^{14} \mathrm{~N},{ }^{22} \mathrm{Na},{ }^{24} \mathrm{Mg},{ }^{26} \mathrm{Al},{ }^{27} \mathrm{Si}$, with a lower rate around $10^{2} \div 10^{3}$ counts/day, contributed to relevant energy depositions in the SVs of the microdosimeter up to $13.3 \mathrm{MeV}$.

Finally, considering the small area of the SOI microdosimeter, we can say that the statistics obtained from our simulation was good to represent the particles scenario encountered inside the ISS. Surely, the microdosimeter will be tested in facilities providing such particles and energies, to evaluate the noise contribution due to the electronics. 

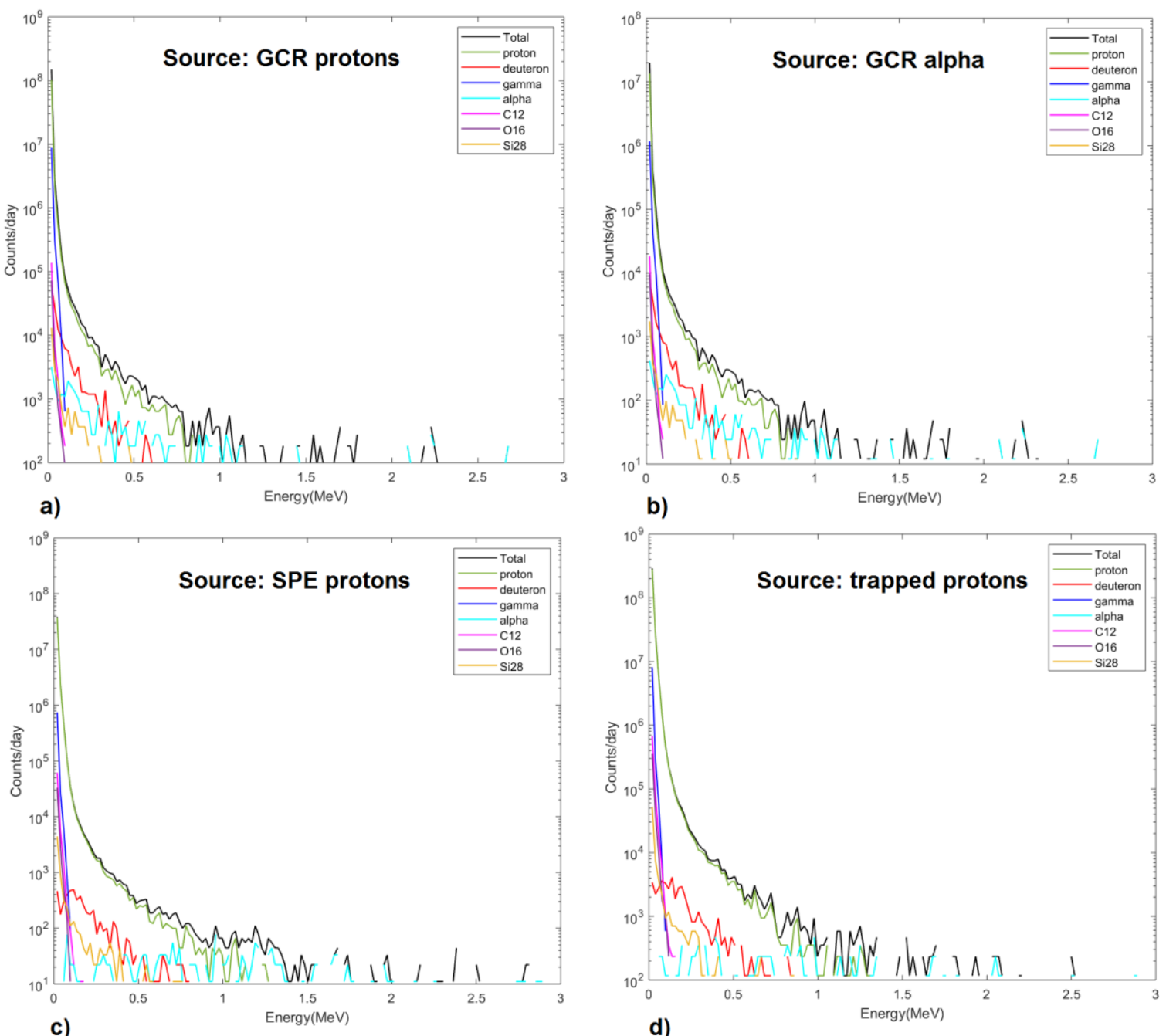

Figure 4. Zoom up to $6 \mathrm{MeV}$ of simulated energy spectra with 1024 channels, representing counts recorded in a 1day mission by the microdosimeter. Four sources were distinguished: a) GCR protons, b) GCR helium nuclei, c) SPE protons, d) Trapped protons.

Figure 5 shows the simulated tissue equivalent microdosimetric spectra obtained with the "3D Mushroom" microdosimeter irradiated with the simulated radiation field inside the Columbus module. The simulations consisted of $2 \cdot 10^{9}$ primary particles for each radiation source (GCR, SPE and trapped protons). The areas of the microdosimetric spectra are normalized to 1 . As expected, both the radiation incident on the ISS (GCR, SPE and trapped protons) and the secondary particles affect the response of the microdosimeter. Protons are mainly responsible for the response corresponding to lineal energies spanning from $0.1 \mathrm{keV} / \mu \mathrm{m}$ to tens of $\mathrm{keV} / \mu \mathrm{m}$. Secondary particles such as electrons, gamma rays, pions, muons are predominant at low lineal energies up to a few $\mathrm{keV} / \mu \mathrm{m}$. It is interesting to see the dose contribution from secondaries produced by GCR protons. A significant peak of secondary helium nuclei around $100 \mathrm{keV} / \mu \mathrm{m}$ originated by GCR protons, accompanied by a few relevant ${ }^{12} \mathrm{C},{ }^{16} \mathrm{O}$ and ${ }^{28} \mathrm{Si}$ ions with lineal energy up to $25 \mathrm{keV} / \mu \mathrm{m}$ with a few exceptions up to $158 \mathrm{keV} / \mu \mathrm{m}$ (Figure $5 \mathrm{a}$ ).

In Figure 5b, microdosimetric spectra from the GCR alpha source show the peak of primary helium nuclei that traverse the Columbus' wall, slowing down in energy due to the interaction and production of secondaries. Again, the production of heavy ions such as ${ }^{12} \mathrm{C},{ }^{16} \mathrm{O}$ and ${ }^{28} \mathrm{Si}$, dominates in the range of lineal energies up to $50 \mathrm{keV} / \mu \mathrm{m}$ with a few ions reaching lineal energy up to $890 \mathrm{keV} / \mu \mathrm{m}$.

Because of their similar energy range (see Figure 5c-d), SPE and trapped protons induce a similar response in the microdosimeter. In both cases, the proton peak is covering the range of lineal energies $0.1 \div 10 \mathrm{keV} / \mu \mathrm{m}$. Secondary heavy ions as ${ }^{12} \mathrm{C},{ }^{16} \mathrm{O},{ }^{28} \mathrm{Si}$ contribute to the microdosimeter response from low lineal energies up to $\sim 150 \mathrm{keV} / \mu \mathrm{m}$ (Figure $5 \mathrm{c}-\mathrm{d}$ ). 

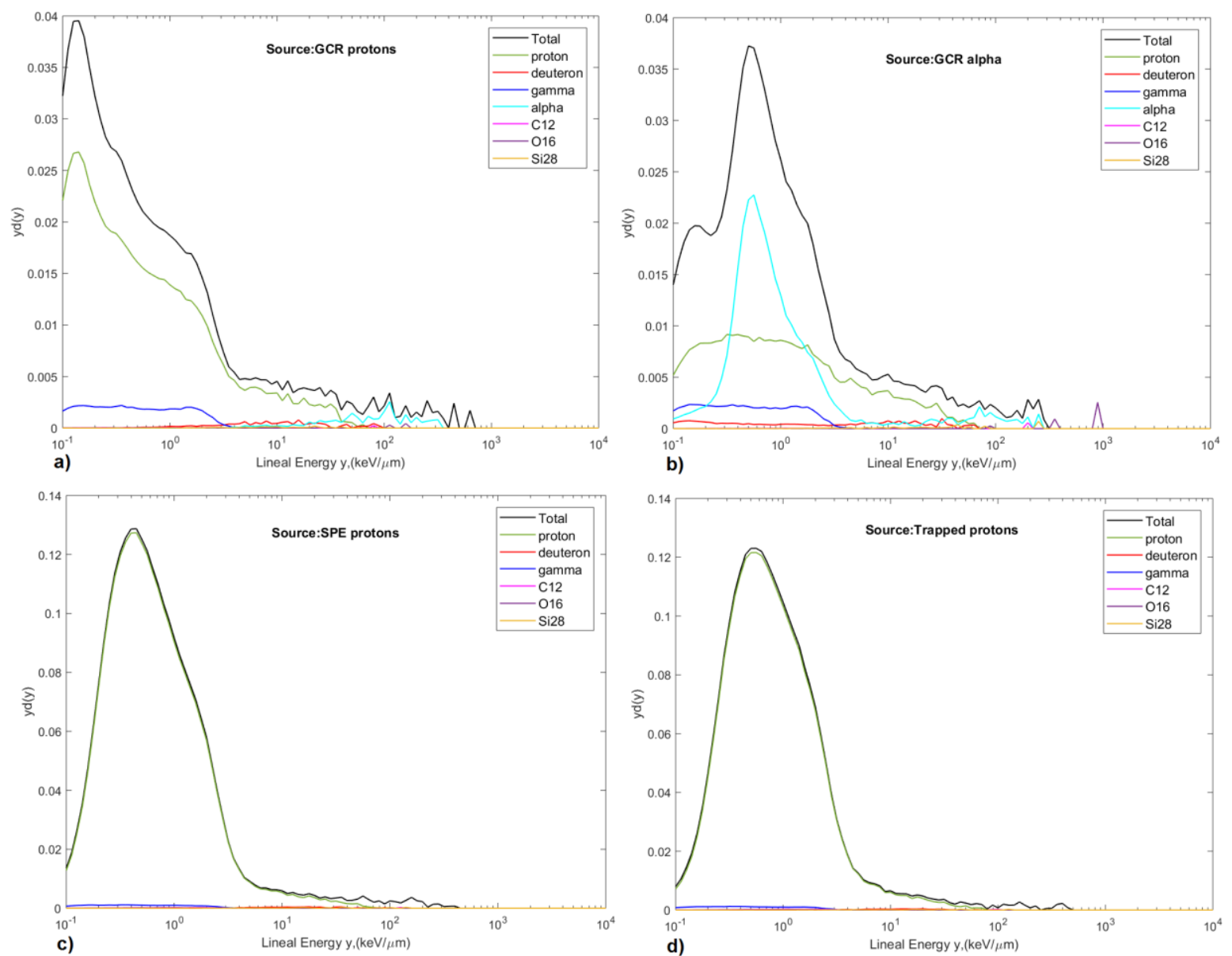

Figure 5. Simulated tissue equivalent microdosimetric spectra as a response of the 3D Mushroom with main radiation sources: a) GCR protons, b) GCR helium nuclei, c) SPE protons, d) Trapped protons. Secondary particles as electrons, pions, muons were detected at low lineal energies. However, their spectra have not been shown in this figure not to overload the graph.

Table 1 shows the values of Absorbed Dose in Tissue Equivalent $D_{T E}$, mean Quality Factor $Q_{m e a n}$ and the corresponding Dose Equivalent $H$ calculated for 1-day mission as explained above.

Observing the microdosimetric spectra in Figure 5c-d for SPE and trapped protons sources, the Q value is lower than 1 because of the predominant contribution to the dose coming from protons with lineal energies below $1 \mathrm{keV} / \mu \mathrm{m}$. On the contrary, for GCR sources the Q value is higher due to low LET protons of high energy that produce target fragments with higher LETs.

Results in terms of dose agree with experimental measurements performed at the ISS's altitude by (L. Sihver and T. Berger et al., 2017) and (T. Dachev et al., 2017) [25, 26]. The highest contribution in terms of dose is due to the trapped protons' source because of the proximity of the inner Van Allen Belt to the ISS's orbit. Moreover, the real-time ISS's orbit calculated by SPENVIS has a quick pass closer to the South Atlantic Anomaly, a region in South America where the inner belt is closer to the Earth's surface, and to the ISS's orbit too, because of the magnetic axis offset of our planet. The $550 \mu \mathrm{Gy} / \mathrm{day}$ of dose deriving from the trapped protons source matches with $567 \mu \mathrm{Gy} /$ day measured by (T. Dachev et al., 2017). The $327 \mu \mathrm{Sv} /$ day of total absorbed dose from GCRs obtained from our simulation respects the value of dose $360 \mu \mathrm{Sv} /$ day for maximum GCRs intensity [26]. Finally, we have not recorded intense SPE events during the day modelled with SPENVIS thus the dose of $42 \mu \mathrm{Gy} /$ day is in the range of doses recorded by (T. Dachev et al., 2017).

According to the choice of adopting a quality factor definition in term of $y$ stated in the ICRU-40, it is clearly justified the result of $Q_{\text {mean }}<1$ for SPE and trapped protons considered as sparsely ionizing radiation sources. Moreover, for completeness and for a direct comaprison to $\mathrm{Q}$ value measured by (T. Dachev et al., 2017), a calculation of $Q(L E T)$ based on ICRP-60 has been done with the knowledge of 
the microdosimetric spectra. Assuming that $y$ is equal to the $L E T$, we obtained a value of $Q_{\text {mean }}$ equal to 1.31 and 1.30 for SPE and trapped protons respectively, which is in agreement with the literature showing a value of Q equal to 1.3 for both sources [26]. This evidence confirms that currently used SOI microdosimeters are capable to measure accurately the quantity y and its distribution in a mixed radiation environment as the one really encountered at the ISS altitude. Nevertheless, depeding on the adopted definition of $\mathrm{Q}$, results can be different for sparsely ionizing radiation. Newer definitions of the quality factor do not invalidate the one stated in ICRU-40, used in this paper. Additionally, (A. M. Kellerer and K. Hahn, 1988) suggested that the option between LET as the reference parameter and the microdosimetric variable, y, may not necessitate an exclusive choice [27].

Table 1. Dose in Tissue Equivalent $\left(D_{T E}\right)$ and Dose Equivalent $(H)$ resulting from the simulation of the microdosimeter embedded in a sphere of $10 \mathrm{~mm}$ radius of PMMA.

\begin{tabular}{ccccc}
\hline Source $2 \pi$ & $\begin{array}{c}\text { Number of particles } \\
(1 \text { day })\end{array}$ & Qmean & $\begin{array}{c}\text { DTE } \\
(\mu \text { Gy/day })\end{array}$ & $\begin{array}{c}\mathrm{H} \\
(\mu \mathrm{Sv} / \text { day })\end{array}$ \\
\hline GCR protons & $1.83 \cdot 10^{11}$ & 1.41 & 190 & 267 \\
GCR alpha & $2.43 \cdot 10^{10}$ & 1.52 & 39 & 60 \\
SPE protons & $2.21 \cdot 10^{10}$ & 0.73 & 42 & 31 \\
Trapped protons & $2.34 \cdot 10^{11}$ & 0.69 & 550 & 378 \\
\hline
\end{tabular}

\section{CONCLUSIONS}

Microdosimetric spectra of GCRs, SPEs, and trapped protons show an evident contribution from protons at low energies, followed by other secondary particles as electrons, gamma rays, helium nuclei, muons. Although the abundance of heavy ions is smaller than protons' one, heavy ions as $\mathrm{C}, \mathrm{O}, \mathrm{Si}, \mathrm{Fe}$ make large contributions to dose, especially because of their high lineal energy.

The absorbed dose and dose equivalent were calculated for a daily mission in space. The good agreement found with data in the literature demonstrates that our Geant 4 model is adequate for simulating the radiation environment at the ISS altitude. In order to better quantify the accuracy of Geant 4 and our simulation application for astronauts' radiation protection, we are currently validating Geant 4 with respect to experimental measurements performed with CMRP microdosimeters at facilities which provide radiation field and heavy ions typically encountered in space, including the CERF facility of CERN (Switzerland) and the HIMAC (Japan).

Moreover, the 3D Mushroom SOI microdosimeter gave an interesting response. Considering the simulation of a 1day mission the counts statistic shows a minimum value of $10^{2}$ counts/channel for each source, at predominant energies. Additionally, the total number of counts spans from $10^{3}$ counts for less frequent particles to $10^{8}$ for protons, each day. This number of counts shows that in principle Mushroom microdosimeters can be used for space radiation protection. Further experiments will test the performance of the microdosimeter to confirm the feasibility against the noise due to the electronics.

In summary, the favourable agreement between the simulated performance of the microdosimeter in the ISS radiation environment against the literature makes the SOI microdosimeter an advantageous candidate to substitute the standard TEPC for dose monitoring in space thanks also to its small dimensions and portability, low voltage supply needed and accuracy in characterizing the field.

It should be noted that for high energy proton field which is part of the spectrum of GCR protons field, the current size of SV SOI microdosimeter will provide smaller $y_{D}$ in comparison with 1um sphere SV of TEPC adopted in $\mathrm{Q}(\mathrm{y})$ dependence. The future work will be devoted to development of SOI microdosimeter with $1 \mathrm{um} \mathrm{SV}$ and reducing the threshold of the measured energy deposited in this SV at the room temperature. 


\section{ACKNOLEDGEMENT}

We acknowledge the CMRP, University of Wollongong that granted the scholarship to carry out this $\mathrm{PhD}$ project, and the European Space Agency (ESA) for supporting the research with the grant "Tissueequivalent crew dosimeter based on novel 3D Si processing”, (Contract No. 4000112670/14/NL/HK).

\section{REFERENCES}

[1] A. Wroe et al., "Microdosimetry simulations of solar protons within a spacecraft," IEEE Transactions on Nuclear Science, vol. 52, no. 6, pp. 2591-2596, 2005.

[2] F. A. Cucinotta and E. Cacao, "Non-Targeted Effects Models Predict Significantly Higher Mars Mission Cancer Risk than Targeted Effects Models," Scientific Reports, vol. 7, no. 1, p. 1832, May 122017.

[3] H. H. Rossi and M. Zaider, Springer, Ed. Microdosimetry and Its Applications. 1996.

[4] P. Bradley, A. Rosenfeld, and M. Zaider, "Solid state microdosimetry," Nuclear Instruments and Methods in Physics Research B, vol. 184, pp. 135-157, 2001.

[5] J. Livingstone et al., "Large Area Silicon Microdosimeter for Dosimetry in High LET Space Radiation Fields: Charge Collection Study," IEEE Transactions on Nuclear Science, vol. 59, no. 6, pp. 3126-3132, 2012.

[6] A. Wroe et al., "Solid State Microdosimetry With Heavy Ions for Space Applications," IEEE Transactions on Nuclear Science, vol. 54, no. 6, pp. 2264-2271, 2007.

[7] D. Prokopovich, M. Reinhard, G. Taylor, A. Hands, and A. Rosenfeld, "Comparison of SOI Microdosimeter and Tissue Equivalent Proportional Counter Measurements at the CERF Facility," IEEE Transactions on Nuclear Science, vol. 59, no. 5, pp. 2501-2505, 2012.

[8] J. Livingstone et al., "Charge Collection in n-SOI Planar Microdosimeters," vol. 60, 2013.

[9] N. Lai, W. Lim, A. Ziebell, M. Reinhard, A. Rosenfeld, and A. Dzurak, "Development and Fabrication of Cylindrical Silicon-on-Insulator Microdosimeter Arrays," IEEE Transactions on Nuclear Science, vol. 56, no. 3, pp. 1637-1641, 2009.

[10] A. Wroe et al., "Silicon Microdosimetry in Heterogeneous Materials: Simulations And Experiements," IEEE TRANSACTIONS ON NUCLEAR SCIENCE, vol. 53, 2006.

[11] T. Ersmark et al., "Geant4 Monte Carlo Simulations of the Belt Proton Radiation Environment On Board the International Space Station/Columbus," IEEE Transactions on Nuclear Science, vol. 54, no. 4, pp. 1444-1453, 2007. ESA. Space Environment Information System (SPENVIS). Available: https://www.spenvis.oma.be/

[13] G. D. BADHWAR and A. KONRADI, "Conversion of omnidirectional proton fluxes into a pitch angle distribution," Journal of Spacecraft and Rockets, vol. 27, no. 3, pp. 350-352, 1990.

[14] D.M. Hassler et al., "The Radiation Assessment Detector (RAD) Investigation," Space Science Reviews, 2012.

[15] R. Destefanis et al., "Space environment characterisation of Kevlar®: good for bullets, debris and radiation too," Universal Journal of Aeronautical \& Aerospace Sciences, vol. 2, pp. 80-113, 2014.

[16] G. Santin, "Normalisation modelling sources," in "Geant4 tutorial," ESA/ESTEC2007.

[17] L. T. Tran et al., "A Novel Silicon Microdosimeter Using 3D Sensitive Volumes: Modeling the Response in Neutron Fields Typical of Aviation," IEEE Transactions on Nuclear Science, vol. 61, no. 4, pp. 1552-1557, 2014.

[18] L. T. Tran et al., "3D-mesa 'bridge' silicon microdosimeter: charge collection study and application to RBE studies in 12C radiation therapy," IEEE Transactions on Nuclear Science, vol. 62, no. 2, 2015.

[19] B. James et al., "SOI Thin Microdosimeter Detectors for Low Energy Ions and Radiation Damage Studies," IEEE Transactions on Nuclear Science, 2018.

[20] D. Bolst et al., "RBE study using solid state microdosimetry in heavy ion therapy," Radiation Measurements, vol. 106, pp. 512-518, 2017.

[21] S. Guatelli et al., "Tissue Equivalence Correction in Silicon Microdosimetry for Protons Characteristic of the LEO Space Environment," IEEE Transactions on Nuclear Science, vol. 55, no. 6, pp. 3407-3413, 2008.

[22] ICRU Report 40, 1986.

[23] ICRP Pubblication 60, 1998.

[24] L. T. Tran et al., "Thin Silicon Microdosimeter Utilizing 3-D MEMS Fabrication Technology: Charge Collection Study and Its Application in Mixed Radiation Fields," IEEE Transactions on Nuclear Science, vol. 65, no. 1, pp. 467-472, 2018.

[25] S. Lembit and T. Berger, "The DOSIS and DOSIS 3D project on-board the ISS - Current status and scientific overview," presented at the IEEE Aerospace Conference, 2016, 2017.

[26] T. Dachev et al., "Overview of the ISS Radiation Environment Observed during the ESA EXPOSE-R2 Mission in 2014-2016," Space Weather, vol. 15, no. 11, pp. 1475-1489, 2017.

[27] A. M. Kellerer and K. Hahn, "Consideration on a revision of the Quality Factor," Radiation Research, vol. 114, 1988. 